\title{
LOW YIELD SPUTTERING OF MONOCRYSTALLINE METALS
}

\author{
A. VAN VEEN ${ }^{*}$ and J.M. FLUIT \\ Physical Laboratory, University Utrecht, Princetonplein 5, The Netherlands
}

\begin{abstract}
Sputtering of monocrystalline metals by light noble gas ions is studied experimentally and theoretically at low primary ion energy. Evidence is found for a multiple collision process in which surface atoms are sputtered by backscattered ions. The introduction of the maximum recoil energy $E_{\mathrm{M}}$ in the sputtering yield relation enabled us to describe sputtering when small energy transfers are involved.
\end{abstract}

\section{Introduction}

The growing interest in the mechanisms involved in first-wall erosion of plasma-fusion reactors has given a new impetus to sputtering studies with light ions [1]. The energies of the projectiles impinging on the first-wall material are in the energy region of $0-20$ $\mathrm{keV}$. Since light projectiles (H, D, T, He) are involved small energy transfers to the atoms of the metal wall (e.g., Mo, W) are encountered. Therefore the maximum recoil energy will be limited to an energy which is about one order of magnitude above the surface binding energy $E_{\mathrm{b}}$ of the metal atoms. In this aspect the sputtering processes are similar to sputtering processes occurring at energies near the sputtering threshold energy for heavier projectiles. The influence of the limitation of the recoil energy is not included in the general sputtering theory developed by Sigmund $[2,3]$. This shows up experimentally for low projectile energies, where the dependence of the sputtering yield deviates strongly from the predicted behaviour.

We present in this paper the results of angular distribution measurements of sputtered particles for a wide range of projectile-target mass ratios $(0.5<$ $\left.M_{2} / M_{1}<10\right)$ and a range of projectile energies from the threshold energy $E_{\mathrm{th}}$ to some tens of $E_{\mathrm{th}}$. These varying conditions enabled us to investigate: (1) the threshold mechanism of sputtering; (2) the influence of the maximum recoil energy; (3) the influence of the projectile-target mass ratio on sputtering.

\footnotetext{
* Present address: Interuniversity Reactor Institute, Mekelweg 15, Delft, The Netherlands.
}

In order to describe the sputtering yield in the low yield region we introduced a sputtering formula which accounts for small energy transfers.

\section{Experiment}

The experiments are carried out in a low pressure gas discharge apparatus [4,5] (e.g., $100 \mathrm{eV} \mathrm{Kr}^{+}$ions: $10^{-2} \mathrm{~Pa}$ of $\mathrm{Kr}$, flux of normal incident $\mathrm{Kr}^{+}$ions $3 \mathrm{~mA} / \mathrm{cm}^{-2}$, space charge sheath 0.5 thick).

The sputtered neutral particles are detected by a rotatable quartz crystal microbalance [5] (polar angle $\vartheta$ from the surface normal variable between 0 and $90^{\circ}$, azimuthal angle $\varphi$ variable by crystal rotation, acceptance angle $3 \times 10^{-2}$ sr). The differentiated frequency change of the crystal is directly proportional to the differential sputtering yield $S(\vartheta, \varphi)$. Calibration was performed using polycrystalline targets (lower detection limit $3 \times 10^{-3}$ atom ion ${ }^{-1} \mathrm{sr}^{-1}$ ).

The average energy of the emitted particles $U(\vartheta, \varphi)$ was measured with the rotatable calorimetric detector designed by Weijsenfeld [4] (acceptance angle $2 X$ $10^{-3} \mathrm{sr}$, lower detection limit $0.1 \mathrm{eV}$ ion $^{-1} \mathrm{sr}^{-1}$ ).

\section{Results for low projectile energies; threshold sputtering mechanism}

We measured the angular distributions of sputtered atoms $S(\vartheta, \varphi)$ and the spatially distributed energy $U(\vartheta, \varphi)$ of sputtered atoms (or backscattered projectiles) for projectile energies down to $20 \mathrm{eV}$. One of the observed features was the existence of the well known sputtering patterns, observed for high projec- 

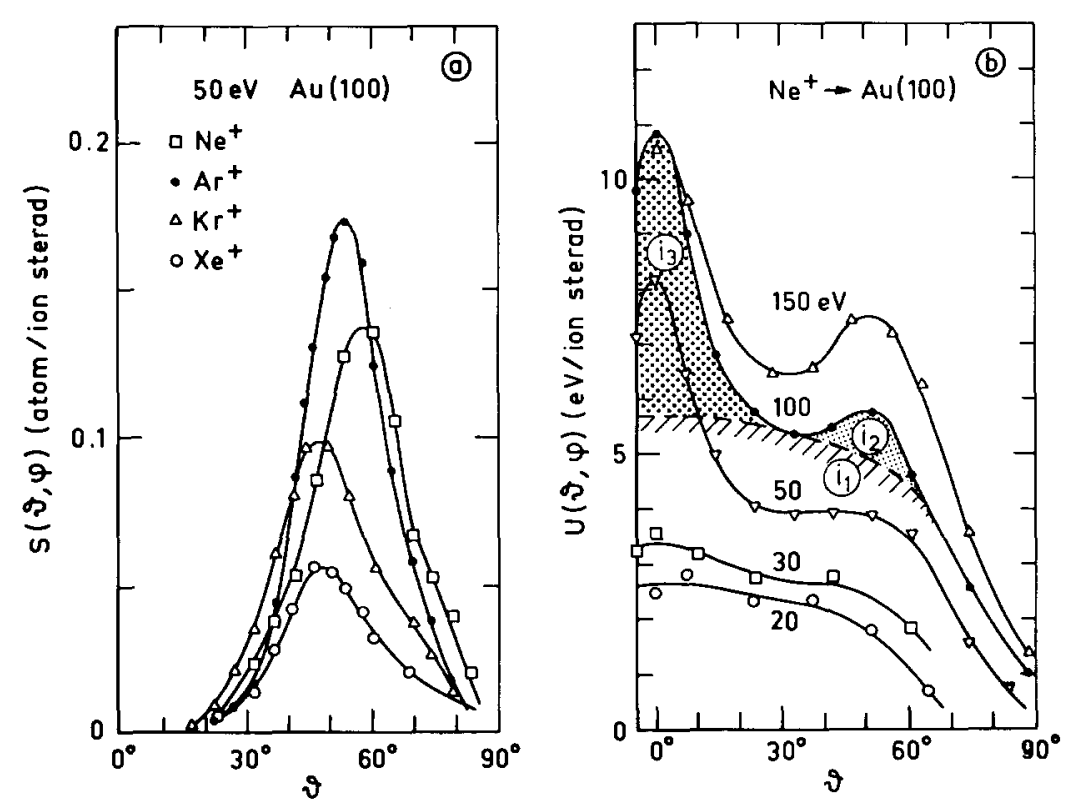

Fig. 1. (a) The differential sputtering yield in an oblique preferential sputtering peak versus the polar ejection angle for $50 \mathrm{eV}$ noble gas ions on $\mathrm{Au}(100)$. (b) The energy transferred to the calorimetric detector (backscattered Ne and sputtered Au) as a function of the polar ejection angle (azimuth [001]) for 20-150 eV Ne${ }^{+}$on $\mathrm{Au}(100)$. Different contributions to the $100 \mathrm{eV}$ curve are indicated by $i_{1}, i_{2}$ and $i_{3}$.

tile energy $(1 \mathrm{keV})$, even at the threshold energy for sputtering. The relative intensities of the sputtering peaks vary drastically in the energy region from $E_{\mathrm{th}}$ to $10 E_{\mathrm{th}}$. In the normal direction we observed a much higher threshold energy than in the oblique directions. Furthermore, we observed for light projectiles $\left(M_{2} / M_{1}>1\right)$ higher sputtering yields than for heavy particles $\left(M_{2} / M_{1}<1\right)$ if the projectile energy was near the threshold energy. Fig. la shows the results of angular distribution measurements of $\mathrm{Ne}$, $\mathrm{Ar}, \mathrm{Kr}, \mathrm{Xe}$ on $\mathrm{Au}(100)$ at $50 \mathrm{eV}$. The observed peak is one of the four oblique preferential sputtering peaks $(\langle 110\rangle)$ typical for fcc $(100)$ surfaces. Deviations in position of the sputtering peak appear for light projectiles.

Measurements of $U(\vartheta, \varphi)$ revealed the especially for light projectiles a large fraction of the energy tranferred to the calorimetric detector cannot be ascribed to sputtered particles, because the angular distributions $U(\vartheta, \varphi)$ and $S(\vartheta, \varphi)$ do not correspond.

In fig. $1 \mathrm{~b}$ we plotted $U(\vartheta, \varphi)$ measured for $\mathrm{Ne} \rightarrow$ $\mathrm{Au}(100)$ for energies down to $20 \mathrm{eV}$. It should be noticed that a rather high energy (due to backscattered projectiles) is measured in the directions in which very few sputtered particles are ejected (see fig. 1a). The measured distributions are very similar to the angular distributions of scattered $\mathrm{Ne}$ on $\mathrm{Au}(100)$ and $\mathrm{Ar}$ on $\mathrm{Cu}(100)$ [5], derived by us and by Smith et al. [6] from trajectory calculations.

The experimental results will be discussed using typical collision processes near threshold. Fig. 2 shows schematically the collision processes in a $\{100\}$ plane of an $f c c(100)$ surface which occur when the surface is bombarded by light projectiles. We found two ways for backscattering to occur. A portion of the Ne particles (impact $i_{3}$ ) is backscattered from subsurface atoms in the Au crystal (atom 3), and another portion is backscattered (impact $i_{1}$ ) from surface atoms. It appears that the trajectories of the particles

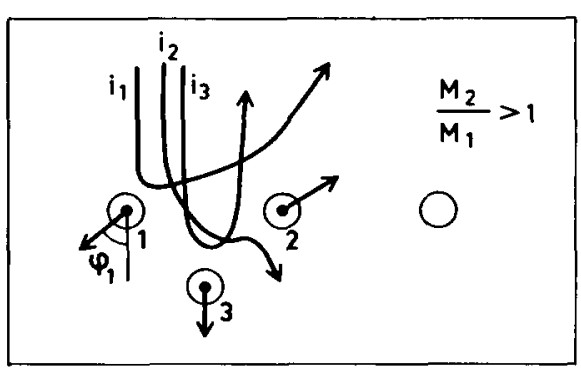

Fig. 2. Collision processes in a (100) crystal surface, caused by bombardment with light $\left(M_{2} / M_{1}>1\right)$ projectiles. 
backscattered from subsurface atoms on their way out are limited by four atoms in the surface layer so that they return close to the normal direction. The directions of the particles backscattered by a single surface atom are limited by collisions with adjacent atoms. The interval of ejection directions, however, is much wider.

We assign the narrow peak at $\vartheta=0^{\circ}$ in fig. $1 \mathrm{~b}$ to Ne particles backscattered from subsurface atoms. The contribution indicated by $i_{1}$ in fig. $1 \mathrm{~b}$ is assigned to particles backscattered from the surface.

Since the results of collision processes caused by projectiles incident at the impact points $i_{1}$ and $i_{3}$ are observed experimentally it is obvious that the intermediate collision process, caused by an impact $i_{2}$ between $i_{1}$ and $i_{3}$ will occur as well. This process leads to sputtering of a surface atom (fig. 2, atom 2) which is hit from below by a backscattered projectile. The contribution in $U(\vartheta, \varphi)$ of the sputtered atoms is found as contribution $i_{2}$ in fig. $1 \mathrm{~b}$.

Heavy projectiles $\left(M_{2} / M_{1}<1\right)$ cannot be scattered over a large angle so that ejection of a recoil atom by backscattering is not observed. After a collision with a sub-surface atom both the projectile and the atom move into the crystal. The heavy projectile as well as the light projectile produce primary recoils which are inwardly directed with an energy $\gamma E \cos ^{2} \varphi_{1}\left[\varphi_{1}\right.$ is the recoil angle; see fig. 2; $\left.\gamma=4 M_{1} M_{2}\left(M_{1}+M_{2}\right)^{-2}\right]$ and may start a collision sequence leading to sputtering. However, we found (section 4) that this process is less efficient than the backscattering process (for light ions).

4. The energy dependence of the differential sputtering yield

Figure 3 shows the differential sputtering yield versus the projectile energy in the normal direction $\left(\vartheta=0^{\circ}\right)$ and in the direction of the oblique sputtering maxima $\left(\vartheta=50^{\circ}\right)$ for all the projectile-target combinations $[(100)$ orientation] we used. For (110) orientated crystals similarly shaped curves are obtained for sputtering in the $\vartheta=0^{\circ}$ and $\vartheta=60^{\circ}$ directions.

In the sputtering theory of Sigmund [3] it is assumed that the recoil atoms have an isotropic distribution; such a distribution causes a cosinelike angular distribution of sputtered atoms. Anisotropy of the flux distribution of the recoil atoms can be observed by deviations from the cosine. In the case of the sputtering of monocrystals we expect that a change of the flux distribution of the recoil atoms will be seen by altered relative intensities of the

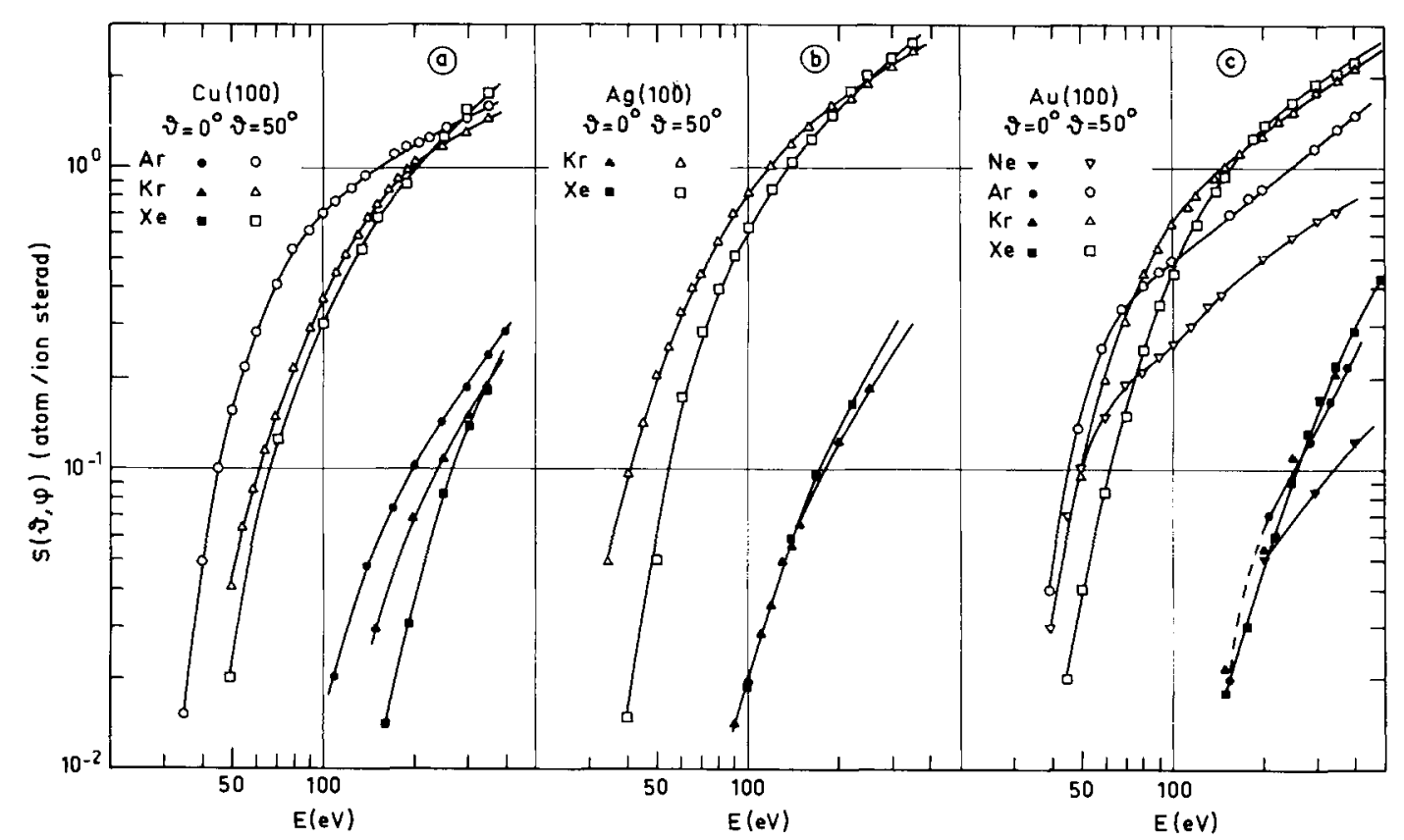

Fig. 3. Differential sputtering yield versus the projectile energy, measured for noble gas ions on (a) $\mathrm{Cu}(100),(\mathrm{b}) \mathrm{Ag}(100)$ and (c) $\mathrm{Au}(100)$ in two directions $\vartheta=0^{\circ}$ and $\vartheta=50^{\circ}$ (peak maximum). The solid lines are to guide the eye. 
sputtering peaks. In fact, we observe that up to 500 $\mathrm{eV}$ projectile energy the intensity ratio of the sputtering yield in the perpendicular direction and in the oblique direction increased (see fig. 3). This implies that at $500 \mathrm{eV}$ "isotropy" is still not attained in the monocrystal. The general trend, found by extrapolating the sputtering curves to higher energy, is that for light projectiles "isotropy" will be attained at a lower projectile energy than for heavy projectiles [compare the extreme cases $M_{2} / M_{1}=0.5(\mathrm{Xe} \rightarrow \mathrm{Cu})$ and $M_{2} / M_{1}$ $=10(\mathrm{Ne} \rightarrow \mathrm{Au})$ in fig. 3]. Measurements for a polycrystalline metal showed the same behaviour. (We should notice that an "isotropic" flux distribution in a monocrystal can be defined as a "symmetric" flux distribution, i.e., symmetric with respect to the low index directions.) Apparently, the observed "anisotropy" is related to the turning of momentum in the single crystal. The maximum recoil energy $E_{M}$ will depend on the direction of the recoil atoms at low energy.

In order to describe the projectile energy dependence of the differential sputtering yield near threshold we assumed an energy spectrum of recoil atoms moving in the direction $\Omega_{0}$

$\mathrm{d} N\left(E_{0}, \Omega_{0}\right) \sim F_{\mathrm{D}}(E)\left[E_{0}^{-2}-E_{\mathrm{M}}\left(E, \Omega_{0}\right)^{-2}\right] \mathrm{d} E_{0} \mathrm{~d} \Omega_{0}$

for $E_{0} \leqslant E_{\mathrm{M}}$ where $F_{\mathrm{D}}(E)$ is the deposited energy in the recoil atoms at the surface [see also the definition of $F_{\mathrm{D}}(E)$ in refs 3 and 4$]$ and $E_{\mathrm{M}}$ is the energy and direction dependent maximum recoil energy. Note that eq. (1) is a modification of the spectrum $E_{0}^{-2} \mathrm{~d} E_{0}$ occurring in cascade theory [3]. The differential sputtering yield in a certain ejection direction $\Omega$ is found by integration of eq. (1) multiplied by the ejection probability $\left(\mathrm{d} \Omega / \mathrm{d} \Omega_{0}\right)$ within the limits $E_{\mathrm{b}}<E_{0}<E_{\mathrm{M}}$.

In general, for single crystal sputtering, the ejection probability is a rather complex function of $E_{\mathrm{b}}, E_{0}$, $\Omega_{0}$ and $\Omega$. However, for sputtering in a preferential sputtering direction this function can be approximated by

$$
\Delta \vartheta^{2} / \Delta \vartheta_{0}^{2}=\Lambda_{0}^{-2}\left(1-E_{\mathrm{b}} / E_{0}\right)^{q}
$$

where $\Delta \vartheta$ and $\Delta \vartheta_{0}$ are the angular deviations of the final and initial directions of the ejected atom, with respect to the (preferential) ejection direction. The factor $\Lambda_{0}$ accounts for the enhancement of the sputtered intensity in preferential directions and the parameter $q$ for refraction effects; e.g., for perpendicular ejection from a (100) surface we take
$\Lambda_{0}=1$ (no preferential sputtering) and $q=1$ giving the usual expression $\left(1-E_{\mathrm{b}} / E_{0}\right)$ following from the uniform potential barrier model for surface ejection. In oblique directions values of $\Lambda_{0}$ and $q$ are found either by surface ejection calculations $[5,8]$ or by fitting of the energy distribution

$\mathrm{d} N\left(E_{\mathrm{s}}\right) \sim\left(1+E_{\mathrm{b}} / E_{\mathrm{s}}\right)^{-q}\left[\left(E_{\mathrm{s}}+E_{\mathrm{b}}\right)^{-2}-E_{\mathrm{M}}^{-2}\right] \mathrm{d} E_{\mathrm{s}}$

of sputtered atoms, which follows from the above assumptions with $E_{0}=E_{\mathrm{s}}+E_{\mathrm{b}}\left(E_{\mathrm{s}}=\right.$ the energy of the sputtered atoms), to experimental energy spectra e.g., measured by Stuart and Wehner [6]. We found generally a close correspondence with the measured curves for $\mathrm{Cu}(100)$ surfaces if $q=1$ substituted for the perpendicular and $q=2$ for the oblique direction, while $E_{\mathrm{M}}(E)$ steadily increases with the projectile energy $E$.

The differential sputtering yield is derived from the proposed expression of the recoil spectrum by integration over $E_{0}\left(E_{\mathrm{b}} \leqslant E_{0} \leqslant E_{\mathrm{M}}\right)$ and gives

$S_{1,2}(E)=c F_{\mathrm{D}}(E) E_{\mathrm{b}}^{-1} \Lambda_{0}^{-2} g_{1,2}\left[E_{\mathrm{b}} / E_{M_{1,2}}(E)\right]$

for the yield in the perpendicular and oblique directions, indicated by the index 1 and 2 respectively, where

$g_{1}(u)=(1-u)^{2}-\frac{1}{2}\left(1-u^{2}\right)-u^{2} \ln u$,

$g_{2}(u)=(1-u)^{2}+2\left(u^{3}-1\right) / 3-2 u^{2} \ln u$,

$c$ is a constant. The maximum recoil energy $E_{\mathrm{M}_{1,2}}$, for normal and oblique incidence of the projectiles respectively, depends on the projectile energy. The curves $g_{1}$ and $g_{2}$ are plotted in fig. $4 \mathrm{a}$. The average energy $\left\langle E_{\mathrm{s}}\right\rangle$ of the sputtered particles can be calculated according to eq. (2). Since $\left\langle E_{s}\right\rangle$ depends on $E_{\mathrm{M}}$ (and $E_{\mathrm{b}}$ ) only, $E_{\mathrm{M}}$ can be found directly from the measured average energy. We were not able to measure accurately the averaged energy at energies near the threshold energy for sputtering owing to the energy contribution of backscattered projectiles. From the average energy given by Stuart and Wehner [6] and measurements by Weijsenfeld at higher energy [4] we derived that $E_{\mathrm{M}}$ first increases linearly with $E$, but at higher energy, if preferential ejection directions are concerned, $E_{\mathrm{M}}$ increases markedly slower.

We tried to match the sputtering yield near the threshold energy by eq. (3) with $E_{\mathrm{M}}=c_{\mathrm{M}} E$ substituted. Since the threshold energy is given in this case by $E_{\mathrm{th}}=E_{\mathrm{b}} / c_{\mathrm{M}}$, it follows that $E_{\mathrm{M}} / E_{\mathrm{b}}=E / E_{\mathrm{th}}$. Further we assumed that the deposited energy $F_{\mathrm{D}}(E)$ was proportional to the projectile energy $E$. There- 

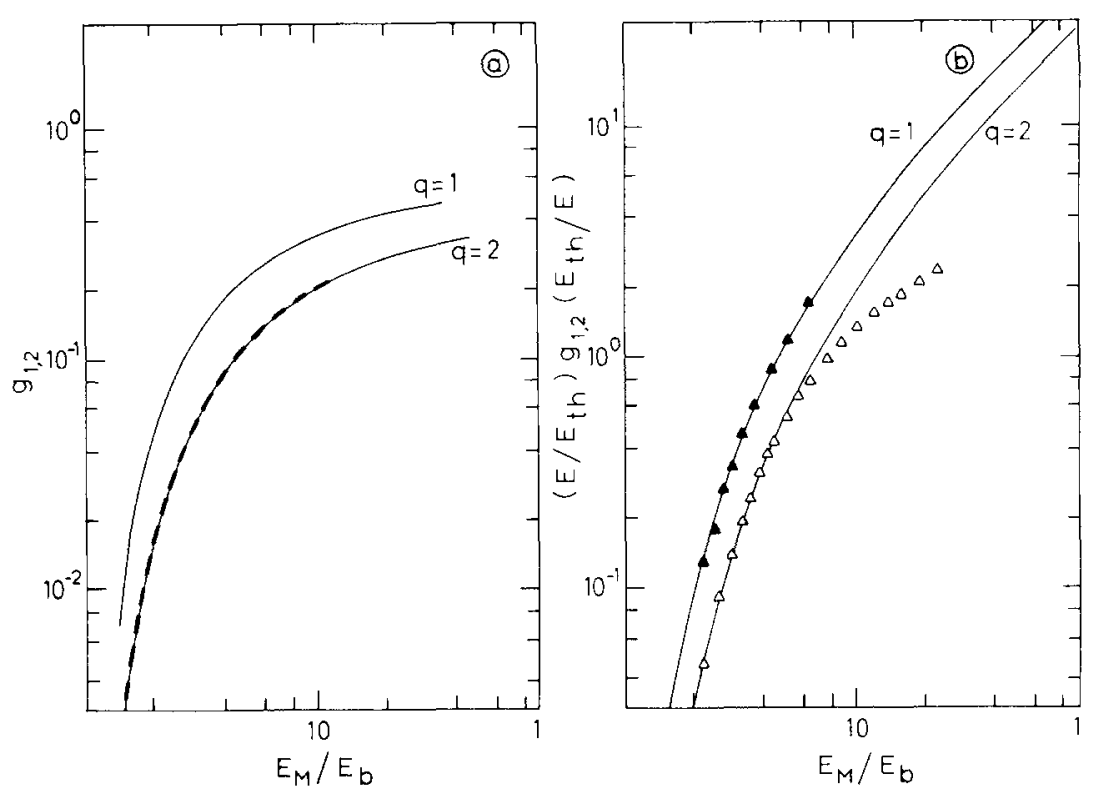

Fig. 4. (a) The yield reduction function $g_{1,2}$ for normal $(q=1)$ and oblique $(q=2)$ ejection. The dashed line represents the universal sputtering curve by Bay et al. [9]. (b) The dependence of the sputtering yield on $E_{\mathrm{M}} / E_{\mathrm{b}}=E / E_{\mathrm{th}}$.

fore

$S_{1,2} \sim E / E_{\mathrm{th}} g_{1,2}\left(E_{\mathrm{th}} / E\right)$.

This is plotted in fig. $4 \mathrm{~b}$. One of the results of the matching procedure $[\mathrm{Kr} \rightarrow \mathrm{Ag}(100)]$ is also shown in fig. $4 \mathrm{~b}$. Further results $\left(c_{M}\right.$ and $\left.E_{\mathrm{th}}\right)$ are given in table 1.

In general the measured sputtering yield curves can be well matched at energies up to $E=80-100 \mathrm{eV}$ for sputtering in oblique directions and up to $E=500$ $\mathrm{eV}$ for sputtering in the perpendicular direction. For sputtering in the oblique direction values of $E_{\mathrm{M}}$ vary from $0.2 E \quad(\mathrm{Xe} \rightarrow \mathrm{Cu})$ to $0.35 E \quad[\mathrm{Ne} \rightarrow \mathrm{Au}(100)]$ and in the perpendicular direction from $0.05 E$ to $0.10 E$ for (100) surfaces and to $0.11 E$ for (110) surfaces. The values for light projectiles are in agree-

Table 1

The threshold parameter $c_{\mathrm{M}}\left(=E_{\mathrm{M}} / E\right.$ ) and the threshold energy $E_{\mathrm{th}}$ (in eV) for sputtering in the perpendicular and oblique direction (accuracy about $5 \%$ ). $E_{\mathrm{b}}$ (in $\mathrm{eV}$ ) for (100) and (110) surfaces respectively: $\mathrm{Cu} 4.2$ and $4.5, \mathrm{Ag} 3.5$ and $3.75, \mathrm{Au} 4.5$ and 4.9 .

\begin{tabular}{|c|c|c|c|c|c|c|c|c|}
\hline \multirow{3}{*}{$\begin{array}{l}\text { Projectile/ } \\
\text { target }\end{array}$} & \multicolumn{4}{|c|}{$(100)$ surface } & \multicolumn{4}{|c|}{ (110) surface } \\
\hline & \multicolumn{2}{|l|}{$\vartheta=0^{\circ}$} & \multicolumn{2}{|c|}{$\vartheta=50^{\circ}$} & \multicolumn{2}{|c|}{$\vartheta=0^{\circ}$} & \multicolumn{2}{|c|}{$\vartheta=60^{\circ}$} \\
\hline & $c_{M}$ & $E_{\mathrm{th}}$ & $c_{M}$ & $E_{\text {th }}$ & $c_{M}$ & $E_{\text {th }}$ & $c_{M}$ & $E_{\text {th }}$ \\
\hline $\mathrm{ArCu}$ & 0.10 & 44 & 0.26 & 17 & 0.12 & 36 & 0.28 & 15 \\
\hline $\mathrm{Kr} \mathrm{Cu}$ & 0.08 & 58 & 0.23 & 20 & 0.11 & 39 & 0.23 & 18 \\
\hline $\mathrm{Xe} \mathrm{Cu}$ & 0.05 & 85 & 0.20 & 23 & 0.10 & 43 & 0.20 & 21 \\
\hline $\mathrm{Kr} \mathrm{Ag}$ & 0.095 & 40 & 0.29 & 13 & 0.13 & 27 & 0.32 & 11 \\
\hline $\mathrm{Xe} \mathrm{Ag}$ & 0.09 & 42 & 0.22 & 17 & 0.13 & 27 & 0.29 & 12 \\
\hline $\mathrm{Ne} \mathrm{Au}$ & - & - & 0.35 & 14 & - & - & 0.28 & 16 \\
\hline $\mathrm{Ar} \mathrm{Au}$ & - & - & 0.31 & 16 & - & - & 0.27 & 17 \\
\hline $\mathrm{Kr} \mathrm{Au}$ & 0.065 & 75 & 0.29 & 17 & 0.10 & 45 & 0.28 & 16 \\
\hline $\mathrm{Xe} \mathrm{Au}$ & 0.06 & 80 & 0.21 & 23 & 0.10 & 45 & 0.24 & 19 \\
\hline
\end{tabular}


ment with calculations of the maximum energy transferable in the backscattering process to a surface recoil atom e.g., $\mathrm{Ne} \rightarrow \mathrm{Au} E_{\mathrm{M}} \simeq 0.33 E$. For a sputtering process via a collision sequence started by the inwardly directed atom 1 (fig. 2) we estimated $E_{\mathrm{M}}<$ $0.1 E$ (assuming $\gamma=1$ ), which may correspond with the threshold mechanism for perpendicular ejection.

The experimental value of $E_{\mathrm{M}}$ for heavy projectiles is in excess of $0.1 E$ and conclusions concerning threshold concerning threshold mechanisms for oblique emission are difficult in that case. We expect that the slow heavy projectile gives rise to a rearrangement of surface atoms during the emission process. This induces lower values for the binding energy.

\section{Application to sputtering by very light ions $\left(M_{2} / M_{1} \gg 1\right)$}

Since very light ions scatter over large angles without much energy loss we expect that the maximum recoil energy will be

$E_{\mathrm{M}} \approx \gamma(1-\gamma) E \approx \gamma E$

in all recoil directions. Consequently the threshold energy $E_{\mathrm{th}}$ is about $E_{\mathrm{b}} / \gamma$ as found experimentally by Bay et al. [9]. Theoretical estimates and computer calculations (MARLOWE) of the surface deposited energy $F_{\mathrm{D}}(E)$ (see Behrisch et al. [10]) show that in the energy range from threshold to several times the threshold energy the deposited energy $F_{\mathrm{D}}(E)$ is a nearly constant function of the projectile energy. Then according to eq. (3) the differential sputtering yield should be proportional to

$g_{1,2}\left(E_{\mathrm{b}} / E_{\mathrm{M}}\right) \equiv g_{1,2}\left(E_{\mathrm{th}} / E\right)$.

The total sputtering yield of a polycrystalline target will be proportional to the sputtering yield in the oblique direction $(q=2)$ because the major part of the sputtered particles (assuming a cosine distribution) is sputtered in oblique directions.

Bay et al. [9] measured the light ion sputtering yield of polycrystalline Au and Mo targets and found that the sputtering yield curves up to $E \approx 10 E_{\mathrm{th}}$ could be normalized to one curve dependent on $E / E_{\mathrm{th}}$ only. Comparison of their normalized sputtering yield curve with $g_{2}\left(E_{\mathrm{th}} / E\right)$ (see fig. 4a) shows a close agreement between both curves. Considering $g_{2}$ as a yield reduction function it seems that the sputtering yield up to very high projectile energies is dominated by threshold effects e.g., for $\mathrm{H}$ on tungsten $E_{\mathrm{M}}<10 E_{\mathrm{b}}$ for proton energies up to $E \approx$ $10 E_{\mathrm{b}} / \gamma=4 \mathrm{keV}$.

A further consequence of the necessary introduction of a yield reduction function for low energy transfers is that the practice [10] of expressing the sputtering yield in the deposited energy $f_{\mathrm{D}}$, (in dimensionless units of the energy $\epsilon$ and depth $\rho$ according to Lindhard et al. [11]) which is independent of the target material, in order to compare analytical values of $f_{\mathrm{D}}$ with values obtained from sputtering measurements, will automatically lead to differences between both values, because the reduction function $g\left(E_{\mathrm{b}} / E_{\mathrm{M}}\right)$ is material dependent. Only at high projectile energies, where $g$ is a constant, one may expect that light ion sputtering yields are proportional to the deposited energy $f_{\mathrm{D}}$.

\section{Final remarks}

It appears that the energy dependence of low energy single crystal sputtering can be described by an empirical sputtering formula which includes an anisotropic maximum recoil energy. In the case of very light ions the proposed sputtering formula is applicable to the description of the experimentally found universal behaviour of the total sputtering yield in the threshold energy region.

This work is part of the research programme of the Foundation for Fundamental Research on Matter (FOM) and was financially supported by the Netherlands Organization for the Advancement of Pure Research (ZWO).

\section{References}

[1] R. Behrisch, in Tokamak reactors for breakeven (Pergamon Press, New York, 1977) p. 37.

[2] P. Sigmund, Phys. Rev. 184 (1969) 383.

[3] P. Sigmund, in Inelastic ion-surface collisions, ed. N.H. Tolk (Academic Press, New York, 1977).

[4] C.H. Weijsenfeld, Thesis, State University Utrecht (1966).

[5] A. van Veen, Thesis, State University Utrecht (1979).

[6] A.G. Smith and G. Carter, Rad. Effects 12 (1972) 63.

[7] R.V. Stuart and G.K. Wehner, J. Appl. Phys. 35 (1963) 1819.

[8] D.P. Jackson, Can. J. Phys. 53 (1975) 1513.

[9] H.L. Bay, J. Roth and J. Bohdansky, J. Appl. Phys. 48 (1977) 4722.

[10] R. Behrisch, G. Maderlechner and B.M.U. Scherzer, Appl. Phys. 18 (1979) 391.

[11] J. Lindhard, N. Scharff and H.E. Schiфtt, Mat. Fys. Medd. Dan. Vid. Selsk. 33 No. 14 (1963). 